\title{
Operations on Generalized Intuitionistic Fuzzy Soft Sets
}

\author{
Jin Han Park \\ Department of Applied Mathematics, Pukyong National University, Busan 608-737, Korea
}

\begin{abstract}
Generalized intuitionistic fuzzy soft set theory, proposed by Park et al. [Journal of Korean Institute of Intelligent Systems 21(3) (2011) 389-394], has been regarded as an effective mathematical tool to deal with uncertainties. In this paper, we prove that certain De Margan's law hold in generalized intuitionistic fuzzy soft set theory with respect to union and intersection operations on generalized intuitionistic fuzzy soft sets. We discuss the basic properties of operations on generalized intuitionistic fuzzy soft sets such as necessity and possibility. Moreover, we illustrate their interconnections between each other.
\end{abstract}

Keywords : Generalized intuitionistic fuzzy soft sets, union, intersection, necessity and possibility operations.

\section{Introduction}

Molodtsov [9] introduced soft sets as a mathematical tool for dealing with uncertainties which is free from the above-mentioned difficulties. Since the soft set theory offers mathematical tool for dealing with uncertain, fuzzy and not clearly defined objects, it has a rich potential for applications to problems in real life situation. The concept and basic properties of soft set theory are presented in $[9,5]$. He also showed how soft set theory is free from the parameterization inadequacy syndrome of fuzzy set theory, rough set theory, probability theory and game theory. However, several assertions presented by Maji et al. [5] are not true in general [1].

Maji et al. [3] presented the concept of fuzzy soft sets which is based on a combination of the fuzzy sets and soft set models. Roy and Maji [14] provided its properties and an application in decision making under imprecise environment. Kong et al. [2] argued that the Roy-Maji method [14] was incorrect and presented a revised algorithm. Zou and Xiao [15] used soft sets and fuzzy soft sets to develop the data analysis approaches under incomplete environment, respectively. Xu et al. [16] introduced the notion of vague soft sets which is an extension to soft sets and is based on a combination of vague sets and soft set models. Majumdar and Samanta [8] further generalized the concept of fuzzy soft sets, in the other words, a degree is attached with the parameterization of fuzzy sets while defining a fuzzy soft set. Maji and his coworker [4, 6, 7] introduced the notion of intuitionistic fuzzy soft set theory which is based on a combination of the intuitionistic fuzzy sets and soft set models

Manuscript received Jul. 20, 2011; revised Aug. 23, 2011; accepted Sep. $6,2011$.

This work was supported by Pukyong National University Research Fund in 2010(PK-2010-034). and studied the properties of intuitionistic fuzzy soft sets. Park et al. [12] presented the concept of the generalized intuitionistic fuzzy soft sets by combining the generalized intuitionistic fuzzy sets [10] and soft set models. Park et al. [13] presented some results on an application of generalized intuitionistic fuzzy soft sets in decision making problems.

In this paper, we try to find an answer to the question how the logic operations and their interrelations between each other correspond to generalized intuitionistic fuzzy soft set operations. The paper is organized as follows: First we prove that a certain De Morgan's law hold in generalized intuitionistic fuzzy soft set theory with respect to union and intersection operations. Then we discuss the basic properties of operations on generalized intuitionistic fuzzy soft sets such as necessity and possibility operations and we illustrate the interconnections between each other. Finally, the conclusion and some topic for future research are presented.

\section{Preliminaries}

In this section we recall some notions related to generalized intuitionistic fuzzy soft sets and some operators on it. See especially $[12,13]$ for further details and background.

Definition 2.1. [12] Let $U$ be an initial universe and $E$ be a set of parameters. $\mathcal{G} \mathcal{I} \mathcal{F}(U)$ denotes the set of all generalized intuitionistic fuzzy sets of $U$. Let $A \subseteq E$. A pair $\langle F, A\rangle$ is a generalized intuitionistic fuzzy soft set over $U$, where $F$ is a mapping given by $F: A \rightarrow \mathcal{G} \mathcal{I} \mathcal{F}(U)$.

In other words, a generalized intuitionistic fuzzy soft set is a parameterized family of generalized intuitionistic fuzzy 
subsets of $U$ and thus its universe is the set of all generalized intuitionistic fuzzy sets of $U$, i.e., $\mathcal{G} \mathcal{I} \mathcal{F}(U)$. A generalized intuitionistic fuzzy soft set is also a special case of a soft set because it is still a mapping from parameters to $\mathcal{G} \mathcal{I} \mathcal{F}(U)$.

Definition 2.2. [12] Let $\langle F, A\rangle$ and $\langle G, B\rangle$ be two generalized intuitionistic fuzzy soft sets over $U$. Then $\langle F, A\rangle$ is said to be a generalized intuitionistic fuzzy soft subset of $\langle G, B\rangle$ if

(1) $A \subseteq B$;

(2) for any $\varepsilon \in A, F(\varepsilon)$ is a generalized fuzzy subset of $G(\varepsilon)$, that is, for all $x \in U$ and $\varepsilon \in A, \mu_{F(\varepsilon)}(x) \leq$ $\mu_{G(\varepsilon)}(x)$ and $\gamma_{F(\varepsilon)}(x) \geq \gamma_{G(\varepsilon)}(x)$.

This relationship is denoted by $\langle F, A\rangle \sqsubseteq\langle G, B\rangle$. Similarly, $\langle F, A\rangle$ is said to be a generalized intuitionistic fuzzy soft superset of $\langle G, B\rangle$, if $\langle G, B\rangle$ is called a generalized intuitionistic fuzzy soft subset of $\langle F, A\rangle$. We denote it by $\langle F, A\rangle \sqsupseteq\langle G, B\rangle$.

Definition 2.3. [12] Let $E=\left\{\varepsilon_{1}, \varepsilon_{2}, \ldots, \varepsilon_{n}\right\}$ be a set of parameters. The not of $E$, denoted by $\rceil E$, is defined by \rceil $E=\left\{\neg \varepsilon_{1}, \neg \varepsilon_{2}, \ldots, \neg \varepsilon_{n}\right\}$, where $\neg \varepsilon_{i}=$ not $\varepsilon_{i}$.

Definition 2.4. [12] The complement of a generalized intuitionistic fuzzy soft set $\langle F, A\rangle$, denoted by $\langle F, A\rangle^{c}$, is defined by $\left.\langle F, A\rangle^{c}=\left\langle F^{c},\right\rceil A\right\rangle$, where $\left.F^{c}:\right\rceil A \rightarrow \mathcal{G I \mathcal { F }}(U)$ is a mapping given by $F^{c}(\varepsilon)=\left\langle x, \gamma_{F(\neg \varepsilon)}(x), \mu_{F(\neg \varepsilon)}(x)\right\rangle$ for all $x \in U$ and $\varepsilon \in\rceil A$.

Definition 2.5. [12] A generalized intuitionistic fuzzy soft set $\langle F, A\rangle$ is said to be a null generalized intuitionistic fuzzy soft set, denoted by $\Phi$, if for any $\varepsilon \in A, \mu_{F(\varepsilon)}(x)=$ 0 and $\gamma_{F(\varepsilon)}(x)=1$, for all $x \in U$.

Definition 2.6. [12] A generalized intuitionistic fuzzy soft set $\langle F, A\rangle$ is said to be an absolute generalized intuitionistic fuzzy soft set, denoted by $\Sigma$, if for any $\varepsilon \in A, \mu_{F(\varepsilon)}(x)=$ 1 and $\gamma_{F(\varepsilon)}(x)=0$, for all $x \in U$.

Definition 2.7. [12] Let $\langle F, A\rangle$ and $\langle G, B\rangle$ be two generalized intuitionistic fuzzy soft sets over a universe $U$. Then " $\langle F, A\rangle$ and $\langle G, B\rangle$ " is a generalized intuitionistic fuzzy soft set, denoted by $\langle F, A\rangle \wedge\langle G, B\rangle$, is defined by $\langle F, A\rangle \wedge\langle G, B\rangle=\langle H, A \times B\rangle$, where $H(\alpha, \beta)=F(\alpha) \cap G(\beta)$ for any $(\alpha, \beta) \in A \times$ $B$, that is, $H(\alpha, \beta)(x)=\left\langle\min \left\{\mu_{F(\alpha)}(x), \mu_{G(\beta)}(x)\right\}\right.$, $\left.\max \left\{\gamma_{F(\alpha)}(x), \gamma_{G(\beta)}(x)\right\}\right\rangle$, for all $(\alpha, \beta) \in A \times B$ and $x \in U$.

Definition 2.8. [12] Let $\langle F, A\rangle$ and $\langle G, B\rangle$ be two generalized intuitionistic fuzzy soft sets over a universe $U$. Then " $\langle F, A\rangle$ or $\langle G, B\rangle$ " is a generalized intuitionistic fuzzy soft set, denoted by $\langle F, A\rangle \vee\langle G, B\rangle$, is defined by $\langle F, A\rangle \vee\langle G, B\rangle=\langle O, A \times B\rangle$, where $O(\alpha, \beta)=F(\alpha) \cup G(\beta)$ for any $(\alpha, \beta) \in A \times$ $B$, that is, $O(\alpha, \beta)(x)=\left\langle\max \left\{\mu_{F(\alpha)}(x), \mu_{G(\beta)}(x)\right\}\right.$, $\left.\min \left\{\gamma_{F(\alpha)}(x), \gamma_{G(\beta)}(x)\right\}\right\rangle$, for all $(\alpha, \beta) \in A \times B$ and $x \in U$.

\section{Operations on generalized intuitionistic fuzzy soft sets}

Definition 3.1. The union of two generalized intuitionistic fuzzy soft sets $\langle F, A\rangle$ and $\langle G, B\rangle$ over a universe $U$ is a generalized intuitionistic fuzzy soft set $\langle H, C\rangle$, where $C=$ $A \cup B$, and for all $\varepsilon \in C$ and $x \in U$,

$$
\begin{array}{r}
\mu_{H(\varepsilon)}(x)= \begin{cases}\mu_{F(\varepsilon)}(x), & \text { if } \varepsilon \in A-B, \\
\mu_{G(\varepsilon)}(x), & \text { if } \varepsilon \in B-A, \\
\max \left\{\mu_{F(\varepsilon)}(x), \mu_{G(\varepsilon)}(x)\right\}, & \text { if } \varepsilon \in A \cap B,\end{cases} \\
\gamma_{H(\varepsilon)}(x)= \begin{cases}\gamma_{F(\varepsilon)}(x), & \text { if } \varepsilon \in A-B, \\
\gamma_{G(\varepsilon)}(x), & \text { if } \varepsilon \in B-A, \\
\min \left\{\gamma_{F(\varepsilon)}(x), \gamma_{G(\varepsilon)}(x)\right\}, & \text { if } \varepsilon \in A \cap B .\end{cases}
\end{array}
$$

We denoted it by $\langle F, A\rangle \sqcup\langle G, B\rangle=\langle H, C\rangle$.

Definition 3.2. The intersection of two generalized intuitionistic fuzzy soft sets $\langle F, A\rangle$ and $\langle G, B\rangle$ over a universe $U$ is a generalized intuitionistic fuzzy soft set $\langle H, C\rangle$, where $C=A \cup B$, and for all $\varepsilon \in C$ and $x \in U$,

$$
\begin{array}{r}
\mu_{H(\varepsilon)}(x)= \begin{cases}\mu_{F(\varepsilon)}(x), & \text { if } \varepsilon \in A-B, \\
\mu_{G(\varepsilon)}(x), & \text { if } \varepsilon \in B-A, \\
\min \left\{\mu_{F(\varepsilon)}(x), \mu_{G(\varepsilon)}(x)\right\}, & \text { if } \varepsilon \in A \cap B,\end{cases} \\
\gamma_{H(\varepsilon)}(x)= \begin{cases}\gamma_{F(\varepsilon)}(x), & \text { if } \varepsilon \in A-B, \\
\gamma_{G(\varepsilon)}(x), & \text { if } \varepsilon \in B-A, \\
\max \left\{\gamma_{F(\varepsilon)}(x), \gamma_{G(\varepsilon)}(x)\right\}, & \text { if } \varepsilon \in A \cap B .\end{cases}
\end{array}
$$

We denoted it by $\langle F, A\rangle \sqcap\langle G, B\rangle=\langle H, C\rangle$.

Theorem 3.3. Let $E$ be a set of parameters and $A \subset E$. If $\Phi$ is a null generalized intuitionistic fuzzy soft set, $\Sigma$ is an absolute generalized intuitionistic fuzzy soft set, and $\langle F, A\rangle$ and $\langle F, E\rangle$ are two generalized intuitionistic fuzzy soft sets over $U$, then the following properties hold:
(1) $\langle F, A\rangle \sqcup\langle F, A\rangle=\langle F, A\rangle$;
(2) $\langle F, A\rangle \sqcap\langle F, A\rangle=\langle F, A\rangle$;
(3) $\langle F, E\rangle \sqcup \Phi=\langle F, E\rangle$;
(4) $\langle F, E\rangle \sqcap \Phi=\Phi$;
(5) $\langle F, E\rangle \sqcup \Sigma=\Sigma$;
(6) $\langle F, E\rangle \sqcap \Sigma=\langle F, E\rangle$.

Proof. It is straightforward from Definitions 3.1 and 3.2.

Theorem 3.4. For two generalized intuitionistic fuzzy soft sets $\langle F, A\rangle$ and $\langle G, B\rangle$ over a universe $U$, the following properties hold:
(1) $(\langle F, A\rangle \sqcup\langle G, B\rangle)^{c}=\langle F, A\rangle^{c} \sqcap\langle G, B\rangle^{c}$;
(2) $(\langle F, A\rangle \sqcap\langle G, B\rangle)^{c}=\langle F, A\rangle^{c} \sqcup\langle G, B\rangle^{c}$.

Proof. (1) Suppose that $\langle F, A\rangle \sqcup\langle G, B\rangle=\langle H, C\rangle$, where $C=A \cup B$, and for all $\varepsilon \in C$ and $x \in U$,

$$
\begin{array}{r}
\mu_{H(\varepsilon)}(x)= \begin{cases}\mu_{F(\varepsilon)}(x), & \text { if } \varepsilon \in A-B, \\
\mu_{G(\varepsilon)}(x), & \text { if } \varepsilon \in B-A, \\
\max \left\{\mu_{F(\varepsilon)}(x), \mu_{G(\varepsilon)}(x)\right\}, & \text { if } \varepsilon \in A \cap B,\end{cases} \\
\gamma_{H(\varepsilon)}(x)= \begin{cases}\gamma_{F(\varepsilon)}(x), & \text { if } \varepsilon \in A-B, \\
\gamma_{G(\varepsilon)}(x), & \text { if } \varepsilon \in B-A, \\
\min \left\{\gamma_{F(\varepsilon)}(x), \gamma_{G(\varepsilon)}(x)\right\}, & \text { if } \varepsilon \in A \cap B .\end{cases}
\end{array}
$$


Since $\langle F, A\rangle \sqcup\langle G, B\rangle=\langle H, C\rangle$, we have $\langle F, A\rangle \sqcup$ $\left.\langle G, B\rangle^{c}=\langle H, C\rangle^{c}=\left\langle H^{c},\right\rceil C\right\rangle$, where $H^{c}(\neg \varepsilon)=$ $\left\langle x, \gamma_{H(\varepsilon)}(x), \mu_{H(\varepsilon)}(x)\right\rangle$ for all $x \in U$ and $\left.\neg \varepsilon \in\right\rceil C=$ \rceil$(A \cup B)=\rceil A \cup\rceil B$. Hence, for all $x \in U$, we have

$\mu_{H^{c}(\neg \varepsilon)}(x)= \begin{cases}\gamma_{F(\varepsilon)}(x), & \text { if } \varepsilon \in A-B, \\ \gamma_{G(\varepsilon)}(x), & \text { if } \varepsilon \in B-A, \\ \min \left\{\gamma_{F(\varepsilon)}(x), \gamma_{G(\varepsilon)}(x)\right\}, & \text { if } \varepsilon \in A \cap B,\end{cases}$
$\gamma_{H^{c}(\neg \varepsilon)}(x)= \begin{cases}\mu_{F(\varepsilon)}(x), & \text { if } \varepsilon \in A-B, \\ \mu_{G(\varepsilon)}(x), & \text { if } \varepsilon \in B-A, \\ \max \left\{\mu_{F(\varepsilon)}(x), \mu_{G(\varepsilon)}(x)\right\}, & \text { if } \varepsilon \in A \cap B .\end{cases}$

Since $\left.\langle F, A\rangle^{c}=\left\langle F^{c},\right\rceil A\right\rangle$ and $\left.\langle G, B\rangle^{c}=\left\langle G^{c},\right\rceil B\right\rangle$, we have $\left.\left.\langle F, A\rangle^{c} \sqcap\langle G, B\rangle^{c}=\left\langle F^{c},\right\rceil A\right\rangle \sqcap\left\langle G^{c},\right\rceil B\right\rangle$. Suppose that $\langle F, A\rangle^{c} \sqcap\langle G, B\rangle^{c}=\langle I, D\rangle$, where $\left.\left.\left.D=\right\rceil C=\right\rceil A \cup\right\rceil B$, and for all $\neg \varepsilon \in D$ and $x \in U$,

$$
\begin{aligned}
& \mu_{I(\neg \varepsilon)}(x) \\
& = \begin{cases}\mu_{F^{c}(\neg \varepsilon)}(x), & \text { if } \neg \varepsilon \in\rceil A-\rceil B, \\
\mu_{G^{c}(\neg \varepsilon)}(x), & \text { if } \neg \varepsilon \in\rceil B-\rceil A, \\
\min \left\{\mu_{F^{c}(\neg \varepsilon)}(x), \mu_{G^{c}(\neg \varepsilon)}(x)\right\}, & \text { if } \neg \varepsilon \in\rceil A \cap\rceil B,\end{cases} \\
& = \begin{cases}\gamma_{F(\varepsilon)}(x), & \text { if } \varepsilon \in A-B, \\
\gamma_{G(\varepsilon)}(x), & \text { if } \varepsilon \in B-A, \\
\min \left\{\gamma_{F(\varepsilon)}(x), \gamma_{G(\varepsilon)}(x)\right\}, & \text { if } \varepsilon \in A \cap B,\end{cases} \\
& \gamma_{I(\neg \varepsilon)}(x) \\
& \begin{array}{l}
= \begin{cases}\gamma_{F^{c}(\neg \varepsilon)}(x), & \text { if } \neg \varepsilon \in\rceil A-\rceil B, \\
\gamma_{G^{c}(\neg \varepsilon)}(x), & \text { if } \neg \varepsilon \in\rceil B-\rceil A, \\
\max \left\{\gamma_{F^{c}(\neg \varepsilon)}(x), \gamma_{G^{c}(\neg \varepsilon)}(x)\right\}, & \text { if } \neg \varepsilon \in\rceil A \cap\rceil B,\end{cases} \\
= \begin{cases}\mu_{F(\varepsilon)}(x), & \text { if } \varepsilon \in A-B, \\
\mu_{G(\varepsilon)}(x), & \text { if } \varepsilon \in B-A, \\
\max \left\{\mu_{F(\varepsilon)}(x), \mu_{G(\varepsilon)}(x)\right\}, & \text { if } \varepsilon \in A \cap B .\end{cases}
\end{array}
\end{aligned}
$$

Therefore, $H^{c}$ and $I$ are the same operators. Thus, $(\langle F, A\rangle \sqcup\langle G, B\rangle)^{c}=\langle F, A\rangle^{c} \sqcap\langle G, B\rangle^{c}$.

(2) Similar to that of (1).

Theorem 3.5. For three generalized intuitionistic fuzzy soft sets $\langle F, A\rangle,\langle G, B\rangle$ and $\langle H, C\rangle$ over a universe $U$, the following properties hold:

(1) $\langle F, A\rangle \sqcap(\langle G, B\rangle \sqcap\langle H, C\rangle)=(\langle F, A\rangle \sqcap\langle G, B\rangle) \sqcap$ $\langle H, C\rangle$;

(2) $\langle F, A\rangle \sqcup(\langle G, B\rangle \sqcup\langle H, C\rangle)=(\langle F, A\rangle \sqcup\langle G, B\rangle) \sqcup$ $\langle H, C\rangle$;

(3) $\langle F, A\rangle \sqcap(\langle G, B\rangle \sqcup\langle H, C\rangle)=(\langle F, A\rangle \sqcap\langle G, B\rangle) \sqcup$ $(\langle F, A\rangle \sqcap\langle H, C\rangle)$;

(4) $\langle F, A\rangle \sqcup(\langle G, B\rangle \sqcap\langle H, C\rangle)=(\langle F, A\rangle \sqcup\langle G, B\rangle) \sqcap$ $(\langle F, A\rangle \sqcup\langle H, C\rangle)$.

Proof. (1) Suppose that $\langle G, B\rangle \sqcap\langle H, C\rangle=\langle I, D\rangle$, where $S=B \cup C$, and for all $\varepsilon \in S$ and $x \in U$,

$$
\begin{aligned}
& \mu_{I(\varepsilon)}(x)= \begin{cases}\mu_{G(\varepsilon)}(x), & \text { if } \varepsilon \in B-C, \\
\mu_{H(\varepsilon)}(x), & \text { if } \varepsilon \in C-B, \\
\min \left\{\mu_{G(\varepsilon)}(x), \mu_{H(\varepsilon)}(x)\right\}, & \text { if } \varepsilon \in B \cap C,\end{cases} \\
& \gamma_{I(\varepsilon)}(x)= \begin{cases}\gamma_{G(\varepsilon)}(x), & \text { if } \varepsilon \in B-C, \\
\gamma_{H(\varepsilon)}(x), & \text { if } \varepsilon \in C-B, \\
\max \left\{\gamma_{G(\varepsilon)}(x), \gamma_{H(\varepsilon)}(x)\right\}, & \text { if } \varepsilon \in B \cap C .\end{cases}
\end{aligned}
$$

Since $\langle F, A\rangle \sqcap(\langle G, B\rangle \sqcap\langle H, C\rangle)=\langle F, A\rangle \sqcap\langle I, D\rangle$, we suppose that $\langle F, A\rangle \sqcap\langle I, D\rangle=\langle J, T\rangle$, where $T=A \cup S=$ $A \cup B \cup C$, and for all $\varepsilon \in T$ and $x \in U$,

$$
\begin{array}{r}
\mu_{J(\varepsilon)}(x) \\
= \begin{cases}\mu_{F(\varepsilon)}(x), & \text { if } \varepsilon \in A-B-C, \\
\mu_{G(\varepsilon)}(x), & \text { if } \varepsilon \in B-C-A, \\
\mu_{H(\varepsilon)}(x), & \text { if } \varepsilon \in C-B-A, \\
\min \left\{\mu_{F(\varepsilon)}(x), \mu_{G(\varepsilon)}(x)\right\}, & \text { if } \varepsilon \in A \cap B-C, \\
\min \left\{\mu_{F(\varepsilon)}(x), \mu_{H(\varepsilon)}(x)\right\}, & \text { if } \varepsilon \in A \cap C-B, \\
\min \left\{\mu_{G(\varepsilon)}(x), \mu_{H(\varepsilon)}(x)\right\}, & \text { if } \varepsilon \in B \cap C-A, \\
\min \left\{\mu_{F(\varepsilon)}(x), \mu_{G(\varepsilon)}(x), \mu_{H(\varepsilon)}(x)\right\}, & \text { if } \varepsilon \in A \cap B \cap C,\end{cases} \\
\gamma_{J(\varepsilon)}(x) \\
= \begin{cases}\gamma_{F(\varepsilon)}(x), & \text { if } \varepsilon \in A-B-C, \\
\gamma_{G(\varepsilon)}(x), & \text { if } \varepsilon \in B-C-A, \\
\gamma_{H(\varepsilon)}(x), & \text { if } \varepsilon \in C-B-A, \\
\max \left\{\gamma_{F(\varepsilon)}(x), \gamma_{G(\varepsilon)}(x)\right\}, & \text { if } \varepsilon \in A \cap B \cap C, \\
\max \left\{\gamma_{F(\varepsilon)}(x), \gamma_{H(\varepsilon)}(x)\right\}, & \text { if } \varepsilon \in B \cap C-B, \\
\max \left\{\gamma_{G(\varepsilon)}(x), \gamma_{H(\varepsilon)}(x)\right\}, & \text { if } \varepsilon \in A \cap B \cap C .\end{cases} \\
\max \left\{\gamma_{F(\varepsilon)}(x), \gamma_{G(\varepsilon)}(x), \gamma_{H(\varepsilon)}(x)\right\},
\end{array}
$$

Assume that $\langle F, A\rangle \sqcap\langle G, B\rangle=\langle K, V\rangle$, where $V=A \cup B$, and for all $\varepsilon \in V$ and $x \in U$,

$$
\begin{aligned}
& \mu_{K(\varepsilon)}(x)= \begin{cases}\mu_{F(\varepsilon)}(x), & \text { if } \varepsilon \in A-B, \\
\mu_{G(\varepsilon)}(x), & \text { if } \varepsilon \in B-A, \\
\min \left\{\mu_{F(\varepsilon)}(x), \mu_{G(\varepsilon)}(x)\right\}, & \text { if } \varepsilon \in A \cap B,\end{cases} \\
& \gamma_{K(\varepsilon)}(x)= \begin{cases}\gamma_{F(\varepsilon)}(x), & \text { if } \varepsilon \in A-B, \\
\gamma_{G(\varepsilon)}(x), & \text { if } \varepsilon \in B-A, \\
\max \left\{\gamma_{F(\varepsilon)}(x), \gamma_{G(\varepsilon)}(x)\right\}, & \text { if } \varepsilon \in A \cap B .\end{cases}
\end{aligned}
$$

Since $(\langle F, A\rangle \sqcap\langle G, B\rangle) \sqcap\langle H, C\rangle=\langle K, V\rangle \sqcap\langle H, C\rangle$, we suppose that $\langle K, V\rangle \sqcap\langle H, C\rangle=\langle L, W\rangle$, where $W=$ $V \cup C=A \cup B \cup C$, and for all $\varepsilon \in W$ and $x \in U$,

$\mu_{L(\varepsilon)}(x)$

$$
= \begin{cases}\mu_{F(\varepsilon)}(x), & \text { if } \varepsilon \in A-B-C, \\ \mu_{G(\varepsilon)}(x), & \text { if } \varepsilon \in B-C-A, \\ \mu_{H(\varepsilon)}(x), & \text { if } \varepsilon \in C-B-A, \\ \min \left\{\mu_{F(\varepsilon)}(x), \mu_{G(\varepsilon)}(x)\right\}, & \text { if } \varepsilon \in A \cap B-C, \\ \min \left\{\mu_{F(\varepsilon)}(x), \mu_{H(\varepsilon)}(x)\right\}, & \text { if } \varepsilon \in B \cap C-B, \\ \min \left\{\mu_{G(\varepsilon)}(x), \mu_{H(\varepsilon)}(x)\right\}, & \text { if } \varepsilon \in A \cap B \cap C, \\ \min \left\{\mu_{F(\varepsilon)}(x), \mu_{G(\varepsilon)}(x), \mu_{H(\varepsilon)}(x)\right\}, & \end{cases}
$$

$\gamma_{L(\varepsilon)}(x)$

$$
= \begin{cases}\gamma_{F(\varepsilon)}(x), & \text { if } \varepsilon \in A-B-C, \\ \gamma_{G(\varepsilon)}(x), & \text { if } \varepsilon \in B-C-A, \\ \gamma_{H(\varepsilon)}(x), & \text { if } \varepsilon \in C-B-A, \\ \max \left\{\gamma_{F(\varepsilon)}(x), \gamma_{G(\varepsilon)}(x)\right\}, & \text { if } \varepsilon \in A \cap B-C, \\ \max \left\{\gamma_{F(\varepsilon)}(x), \gamma_{H(\varepsilon)}(x)\right\}, & \text { if } \varepsilon \in A \cap C-B, \\ \max \left\{\gamma_{G(\varepsilon)}(x), \gamma_{H(\varepsilon)}(x)\right\}, & \text { if } \varepsilon \in B \cap C-A, \\ \max \left\{\gamma_{F(\varepsilon)}(x), \gamma_{G(\varepsilon)}(x), \gamma_{H(\varepsilon)}(x)\right\}, & \text { if } \varepsilon \in A \cap B \cap C .\end{cases}
$$

Therefore, $\mu_{L(\varepsilon)}(x)=\mu_{J(\varepsilon)}(x)$ and $\gamma_{L(\varepsilon)}(x)=\gamma_{J(\varepsilon)}(x)$ for all $\varepsilon \in A \cup B \cup C$ and $x \in U$. That is, $L$ and $J$ are the same operators. Thus, $\langle F, A\rangle \sqcap(\langle G, B\rangle \sqcap\langle H, C\rangle)=$ $(\langle F, A\rangle \sqcap\langle G, B\rangle) \sqcap\langle H, C\rangle$.

(2)-(4) are proved analogously.

Definition 3.6. The necessity operation on a generalized intuitionistic fuzzy soft set $\langle F, A\rangle$ is denoted by $\odot\langle F, A\rangle$ and is defined as $\odot\langle F, A\rangle=\left\{\left\langle x, \mu_{\odot F(\varepsilon)}(x), \gamma_{\odot F(\varepsilon)}(x)\right\rangle\right.$ : $x \in U, \varepsilon \in A\}$, where $\mu_{\odot F(\varepsilon)}(x)=\mu_{F(\varepsilon)}(x)$ is the generalized intuitionistic fuzzy membership degree that object $x$ holds on parameter $\varepsilon, \gamma_{\odot F(\varepsilon)}(x)=1-\mu_{F(\varepsilon)}(x)$ is the 
generalized intuitionistic fuzzy membership degree that object $x$ does not hold on parameter $\varepsilon$, and $F$ is a mapping $F: A \rightarrow \mathcal{G I F}(U)$.

Theorem 3.7. For two generalized intuitionistic fuzzy soft sets $\langle F, A\rangle$ and $\langle G, B\rangle$ over a universe $U$, the following properties hold:

(1) $\odot(\langle F, A\rangle \sqcup\langle G, B\rangle)=\odot\langle F, A\rangle \sqcup \odot\langle G, B\rangle$;

(2) $\odot(\langle F, A\rangle \sqcap\langle G, B\rangle)=\odot\langle F, A\rangle \sqcap \odot\langle G, B\rangle$;

(3) $\odot \odot\langle F, A\rangle=\odot\langle F, A\rangle$.

Proof. (1) Suppose that $\langle F, A\rangle \sqcup\langle G, B\rangle=\langle H, C\rangle$, where $C=A \cup B$ and for all $\varepsilon \in C$,

$$
\begin{aligned}
& \mu_{H(\varepsilon)}(x)=\left\{\begin{array}{lr}
\mu_{F(\varepsilon)}(x), & \text { if } \varepsilon \in A-B, \\
\mu_{G(\varepsilon)}(x), & \text { if } \varepsilon \in B-A, \\
\max \left\{\mu_{F(\varepsilon)}(x), \mu_{G(\varepsilon)}(x)\right\}, & \text { if } \varepsilon \in A \cap B,
\end{array}\right. \\
& \gamma_{H(\varepsilon)}(x)=\left\{\begin{array}{lr}
\gamma_{F(\varepsilon)}(x), & \text { if } \varepsilon \in A-B, \\
\gamma_{G(\varepsilon)}(x), & \text { if } \varepsilon \in B-A, \\
\min \left\{\gamma_{F(\varepsilon)}(x), \gamma_{G(\varepsilon)}(x)\right\}, & \text { if } \varepsilon \in A \cap B .
\end{array}\right.
\end{aligned}
$$

Since $\odot(\langle F, A\rangle \sqcup\langle G, B\rangle)=\odot\langle H, C\rangle$, by Definition 3.6, we have

$$
\begin{aligned}
& \mu_{\odot H(\varepsilon)}(x) \\
& = \begin{cases}\mu_{F(\varepsilon)}(x), & \text { if } \varepsilon \in A-B, \\
\mu_{G(\varepsilon)}(x), & \text { if } \varepsilon \in B-A, \\
\max \left\{\mu_{F(\varepsilon)}(x), \mu_{G(\varepsilon)}(x)\right\}, & \text { if } \varepsilon \in A \cap B,\end{cases} \\
& \gamma_{\odot H(\varepsilon)}(x) \\
& = \begin{cases}1-\mu_{F(\varepsilon)}(x), & \text { if } \varepsilon \in A-B, \\
1-\mu_{G(\varepsilon)}(x), & \text { if } \varepsilon \in B-A, \\
1-\max \left\{\mu_{F(\varepsilon)}(x), \mu_{G(\varepsilon)}(x)\right\}, & \text { if } \varepsilon \in A \cap B,\end{cases}
\end{aligned}
$$

for all $\varepsilon \in C=A \cup B$ and $x \in U$. Assume that $\odot\langle F, A\rangle=\left\{\left\langle x, \mu_{F(\varepsilon)}(x), 1-\mu_{F(\varepsilon)}(x)\right\rangle: x \in U, \varepsilon \in A\right\}$ and $\odot\langle G, B\rangle=\left\{\left\langle x, \mu_{G(\varepsilon)}(x), 1-\mu_{G(\varepsilon)}(x)\right\rangle: x \in U, \varepsilon \in\right.$ $B\}$. Suppose that $\odot\langle F, A\rangle \sqcup \odot\langle G, B\rangle=\langle O, C\rangle$, where $C=A \cup B$, and for all $\varepsilon \in C$ and $x \in U$,

$$
\begin{aligned}
& \mu_{O(\varepsilon)}(x) \\
& = \begin{cases}\mu_{F(\varepsilon)}(x), & \text { if } \varepsilon \in A-B, \\
\mu_{G(\varepsilon)}(x), & \text { if } \varepsilon \in B-A, \\
\max \left\{\mu_{F(\varepsilon)}(x), \mu_{G(\varepsilon)}(x)\right\}, & \text { if } \varepsilon \in A \cap B,\end{cases} \\
& \gamma_{O(\varepsilon)}(x) \\
& = \begin{cases}1-\mu_{F(\varepsilon)}(x), & \text { if } \varepsilon \in A-B, \\
1-\mu_{G(\varepsilon)}(x), & \text { if } \varepsilon \in B-A, \\
\min \left\{1-\mu_{F(\varepsilon)}(x), 1-\mu_{G(\varepsilon)}(x)\right\}, & \text { if } \varepsilon \in A \cap B,\end{cases} \\
& = \begin{cases}1-\mu_{F(\varepsilon)}(x), & \text { if } \varepsilon \in A-B, \\
1-\mu_{G(\varepsilon)}(x), & \text { if } \varepsilon \in B-A, \\
1-\max \left\{\mu_{F(\varepsilon)}(x), \mu_{G(\varepsilon)}(x)\right\}, & \text { if } \varepsilon \in A \cap B .\end{cases}
\end{aligned}
$$

Consequently, $\odot\langle H, C\rangle$ and $\langle O, C\rangle$ are the same generalized intuitionistic fuzzy soft sets. Thus, $\odot(\langle F, A\rangle \sqcup$ $\langle G, B\rangle)=\odot\langle F, A\rangle \sqcup \odot\langle G, B\rangle$.

(2) and (3) are proved analogously.

Definition 3.8. The possibility operation on a generalized intuitionistic fuzzy soft set $\langle F, A\rangle$ is denoted by $\diamond\langle F, A\rangle$ and is defined as $\diamond\langle F, A\rangle=\left\{\left\langle x, \mu_{\diamond F(\varepsilon)}(x), \gamma_{\diamond F(\varepsilon)}(x)\right\rangle\right.$ : $x \in U, \varepsilon \in A\}$, where $\mu_{\diamond F(\varepsilon)}(x)=1-\gamma_{F(\varepsilon)}(x)$ is the generalized intuitionistic fuzzy membership degree that object $x$ holds on parameter $\varepsilon, \gamma_{\diamond F(\varepsilon)}(x)=\gamma_{F(\varepsilon)}(x)$ is the generalized intuitionistic fuzzy membership degree that object $x$ does not hold on parameter $\varepsilon$, and $F$ is a mapping $F: A \rightarrow \mathcal{G} \mathcal{I} \mathcal{F}(U)$.

Theorem 3.9. For two generalized intuitionistic fuzzy soft sets $\langle F, A\rangle$ and $\langle G, B\rangle$ over a universe $U$, the following properties hold:
(1) $\diamond(\langle F, A\rangle \sqcup\langle G, B\rangle)=\diamond\langle F, A\rangle \sqcup \diamond\langle G, B\rangle$;
(2) $\diamond(\langle F, A\rangle \sqcap\langle G, B\rangle)=\diamond\langle F, A\rangle \sqcap \diamond\langle G, B\rangle$;
(3) $\diamond \diamond\langle F, A\rangle=\diamond\langle F, A\rangle$.

Proof. (1) Suppose that $\langle F, A\rangle \sqcup\langle G, B\rangle=\langle H, C\rangle$, where $C=A \cup B$, and for all $\varepsilon \in C$ and $x \in U$,

$$
\begin{array}{r}
\mu_{H(\varepsilon)}(x)=\left\{\begin{array}{lr}
\mu_{F(\varepsilon)}(x), & \text { if } \varepsilon \in A-B, \\
\mu_{G(\varepsilon)}(x), & \text { if } \varepsilon \in B-A, \\
\max \left\{\mu_{F(\varepsilon)}(x), \mu_{G(\varepsilon)}(x)\right\}, & \text { if } \varepsilon \in A \cap B,
\end{array}\right. \\
\gamma_{H(\varepsilon)}(x)=\left\{\begin{array}{lr}
\gamma_{F(\varepsilon)}(x), & \text { if } \varepsilon \in A-B, \\
\gamma_{G(\varepsilon)}(x), & \text { if } \varepsilon \in B-A, \\
\min \left\{\gamma_{F(\varepsilon)}(x), \gamma_{G(\varepsilon)}(x)\right\}, & \text { if } \varepsilon \in A \cap B .
\end{array}\right.
\end{array}
$$

Since $\diamond(\langle F, A\rangle \sqcup\langle G, B\rangle)=\diamond\langle H, C\rangle$, by Definition 3.8, we have

$$
\begin{aligned}
& \mu_{\diamond H(\varepsilon)}(x) \\
& = \begin{cases}1-\gamma_{F(\varepsilon)}(x), & \text { if } \varepsilon \in A-B, \\
1-\gamma_{G(\varepsilon)}(x), & \text { if } \varepsilon \in B-A, \\
1-\min \left\{\gamma_{F(\varepsilon)}(x), \gamma_{G(\varepsilon)}(x)\right\}, & \text { if } \varepsilon \in A \cap B,\end{cases} \\
& \gamma_{\diamond H(\varepsilon)}(x) \\
& = \begin{cases}\gamma_{F(\varepsilon)}(x), & \text { if } \varepsilon \in A-B, \\
\gamma_{G(\varepsilon)}(x), & \text { if } \varepsilon \in B-A, \\
\min \left\{\gamma_{F(\varepsilon)}(x), \gamma_{G(\varepsilon)}(x)\right\}, & \text { if } \varepsilon \in A \cap B,\end{cases}
\end{aligned}
$$

for all $\varepsilon \in C=A \cup B$ and $x \in U$. Assume that $\diamond\langle F, A\rangle=\left\{\left\langle x, 1-\gamma_{F(\varepsilon)}(x), \gamma_{F(\varepsilon)}(x)\right\rangle: x \in U, \varepsilon \in A\right\}$ and $\diamond\langle G, B\rangle=\left\{\left\langle x, 1-\gamma_{G(\varepsilon)}(x), \gamma_{G(\varepsilon)}(x)\right\rangle: x \in U, \varepsilon \in\right.$ $B\}$. Suppose that $\diamond\langle F, A\rangle \sqcup \diamond\langle G, B\rangle=\langle O, C\rangle$, where $C=A \cup B$, and for all $\varepsilon \in C$ and $x \in U$,

$\mu_{O(\varepsilon)}(x)$

$$
\begin{aligned}
& =\left\{\begin{array}{lr}
1-\gamma_{F(\varepsilon)}(x), & \text { if } \varepsilon \in A-B, \\
1-\gamma_{G(\varepsilon)}(x), & \text { if } \varepsilon \in B-A, \\
\max \left\{1-\gamma_{F(\varepsilon)}(x), 1-\gamma_{G(\varepsilon)}(x)\right\}, & \text { if } \varepsilon \in A \cap B,
\end{array}\right. \\
& = \begin{cases}1-\gamma_{F(\varepsilon)}(x), & \text { if } \varepsilon \in A-B, \\
1-\gamma_{G(\varepsilon)}(x), & \text { if } \varepsilon \in B-A, \\
1-\min \left\{\gamma_{F(\varepsilon)}(x), \gamma_{G(\varepsilon)}(x)\right\}, & \text { if } \varepsilon \in A \cap B,\end{cases} \\
& (x) \quad \begin{array}{ll}
\gamma_{F(\varepsilon)}(x), & \text { if } \varepsilon \in A-B, \\
\gamma_{G(\varepsilon)}(x), & \text { if } \varepsilon \in B-A, \\
\min \left\{\gamma_{F(\varepsilon)}(x), \gamma_{G(\varepsilon)}(x)\right\}, & \text { if } \varepsilon \in A \cap B .
\end{array}
\end{aligned}
$$$$
\gamma_{O(\varepsilon)}(x)
$$

Consequently, $\diamond\langle H, C\rangle$ and $\langle O, C\rangle$ are he same generalized intuitionistic fuzzy soft sets. Thus, $\diamond(\langle F, A\rangle \sqcup$ $\langle G, B\rangle)=\diamond\langle F, A\rangle \sqcup \diamond\langle G, B\rangle$.

(2) and (3) are proved analogously. 
Theorem 3.10. For a generalized intuitionistic fuzzy soft sets $\langle F, A\rangle$ over a universe $U$ with $\langle F, A\rangle=$ $\left\{\left\langle x, \mu_{F(\varepsilon)}(x), \gamma_{F(\varepsilon)}(x)\right\rangle: x \in U, \varepsilon \in A\right\}$, the following properties hold:

(1) $\odot\langle F, A\rangle \sqsubseteq\langle F, A\rangle \sqsubseteq \diamond\langle F, A\rangle$ if $\mu_{F(\varepsilon)}(x)+$ $\gamma_{F(\varepsilon)}(x) \leq 1$ for all $\varepsilon \in A$ and $x \in U ; \odot\langle F, A\rangle \sqsupseteq$ $\langle F, A\rangle \sqsupseteq \diamond\langle F, A\rangle$ if $\mu_{F(\varepsilon)}(x)+\gamma_{F(\varepsilon)}(x) \geq 1$ for all $\varepsilon \in A$ and $x \in U$;

(2) $\diamond \odot\langle F, A\rangle=\odot\langle F, A\rangle$;

(3) $\odot \diamond\langle F, A\rangle=\diamond\langle F, A\rangle$.

Proof. Suppose that $\langle F, A\rangle=\left\{\left\langle x, \mu_{F(\varepsilon)}(x), \gamma_{F(\varepsilon)}(x)\right\rangle\right.$ : $x \in U, \varepsilon \in A\}$. Then,

$$
\begin{aligned}
& \odot\langle F, A\rangle=\left\{\left\langle x, \mu_{F(\varepsilon)}(x), 1-\mu_{F(\varepsilon)}(x)\right\rangle: x \in U, \varepsilon \in A\right\}, \\
& \diamond\langle F, A\rangle=\left\{\left\langle x, 1-\gamma_{F(\varepsilon)}(x), \gamma_{F(\varepsilon)}(x)\right\rangle: x \in U, \varepsilon \in A\right\} .
\end{aligned}
$$

(1) When $\mu_{F(\varepsilon)}(x)+\gamma_{F(\varepsilon)}(x) \leq 1$ for all $\varepsilon \in A$ and $x \in$ $U$, then we have $\mu_{F(\varepsilon)}(x) \leq 1-\gamma_{F(\varepsilon)}(x)$ and $\gamma_{F(\varepsilon)}(x) \leq$ $1-\mu_{F(\varepsilon)}(x)$. Hence $\odot\langle F, A\rangle \sqsubseteq\langle F, A\rangle \sqsubseteq \diamond\langle F, A\rangle$.

When $\mu_{F(\varepsilon)}(x)+\gamma_{F(\varepsilon)}(x) \geq 1$ for all $\varepsilon \in A$ and $x \in U$, then we have $\mu_{F(\varepsilon)}(x) \geq 1-\gamma_{F(\varepsilon)}(x)$ and $\gamma_{F(\varepsilon)}(x) \geq$ $1-\mu_{F(\varepsilon)}(x)$. Hence $\odot\langle F, A\rangle \sqsupseteq\langle F, A\rangle \sqsupseteq \diamond\langle F, A\rangle$.

(2) Since $\odot\langle F, A\rangle=\left\{\left\langle x, \mu_{F(\varepsilon)}(x), 1-\mu_{F(\varepsilon)}(x)\right\rangle: x \in\right.$ $U, \varepsilon \in A\}$, we have

$$
\begin{aligned}
\diamond \odot & \langle F, A\rangle \\
& =\left\{\left\langle x, 1-\left(1-\mu_{F(\varepsilon)}(x)\right), 1-\mu_{F(\varepsilon)}(x)\right\rangle: x \in U, \varepsilon \in A\right\} \\
& =\left\{\left\langle x, \mu_{F(\varepsilon)}(x), 1-\mu_{F(\varepsilon)}(x)\right\rangle: x \in U, \varepsilon \in A\right\} \\
& =\odot\langle F, A\rangle .
\end{aligned}
$$

(3) The proof is similar to that of (2).

Theorem 3.11. For two generalized intuitionistic fuzzy soft sets $\langle F, A\rangle$ and $\langle G, B\rangle$ over a universe $U$, the following properties hold:

(1) $\odot(\langle F, A\rangle \wedge\langle G, B\rangle)=\odot\langle F, A\rangle \wedge \odot\langle G, B\rangle$;

(2) $\odot(\langle F, A\rangle \vee\langle G, B\rangle)=\odot\langle F, A\rangle \vee \odot\langle G, B\rangle$;

(3) $\diamond(\langle F, A\rangle \wedge\langle G, B\rangle)=\diamond\langle F, A\rangle \wedge \diamond\langle G, B\rangle$;

(4) $\diamond(\langle F, A\rangle \vee\langle G, B\rangle)=\diamond\langle F, A\rangle \vee \diamond\langle G, B\rangle$.

Proof. (1) Suppose that $\langle F, A\rangle \wedge\langle G, B\rangle=\langle H$, $A \times B\rangle$, where $H(\alpha, \beta)=F(\alpha) \cap H(\beta)$ for all $(\alpha, \beta) \in A \times B$, that is, $H(\alpha, \beta)(x)=$ $\left\langle\min \left\{\mu_{F(\alpha)}(x), \mu_{G(\beta)}(x)\right\}, \max \left\{\gamma_{F(\alpha)}(x), \gamma_{G(\beta)}(x)\right\}\right\rangle$, for all $(\alpha, \beta) \in A \times B$ and $x \in U$. Thus,

$$
\begin{aligned}
\langle F, A\rangle \wedge & \langle G, B\rangle \\
= & \left\{\left\langlex, \min \left\{\mu_{F(\alpha)}(x), \mu_{G(\beta)}(x)\right\},\right.\right. \\
& \left.\left.\max \left\{\gamma_{F(\alpha)}(x), \gamma_{G(\beta)}(x)\right\}\right\rangle: x \in U,(\alpha, \beta) \in A \times B\right\} .
\end{aligned}
$$

By Definition 3.8, we have

$$
\begin{aligned}
\odot(\langle F, A\rangle & \wedge\langle G, B\rangle) \\
= & \left\{\left\langlex, \min \left\{\mu_{F(\alpha)}(x), \mu_{G(\beta)}(x)\right\}, 1-\right.\right. \\
& \left.\left.\min \left\{\mu_{F(\alpha)}(x), \mu_{G(\beta)}(x)\right\}\right\rangle: x \in U,(\alpha, \beta) \in A \times B\right\} .
\end{aligned}
$$

Since $\odot\langle F, A\rangle=\left\{\left\langle x, \mu_{F(\alpha)}(x), 1-\mu_{F(\alpha)}(x)\right\rangle: x \in\right.$ $U, \alpha \in A\}$ and $\odot\langle G, B\rangle=\left\{\left\langle x, \mu_{G(\beta)}(x), 1-\mu_{G(\beta)}(x)\right\rangle\right.$ : $x \in U, \beta \in B\}$, we have

$$
\begin{aligned}
& \odot\langle F, A\rangle \wedge \odot\langle G, B\rangle \\
&=\left\{\left\langlex, \min \left\{\mu_{F(\alpha)}(x), \mu_{G(\beta)}(x)\right\}, \max \left\{1-\mu_{F(\alpha)}(x),\right.\right.\right. \\
&\left.\left.\left.1-\mu_{G(\beta)}(x)\right\}\right\rangle: x \in U,(\alpha, \beta) \in A \times B\right\} \\
&=\left\{\left\langlex, \min \left\{\mu_{F(\alpha)}(x), \mu_{G(\beta)}(x)\right\}, 1-\right.\right. \\
&\left.\left.\min \left\{\mu_{F(\alpha)}(x), \mu_{G(\beta)}(x)\right\}\right\rangle: x \in U,(\alpha, \beta) \in A \times B\right\} \\
&= \odot(\langle F, A\rangle \wedge\langle G, B\rangle) .
\end{aligned}
$$

(2) The proof is similar to that of (1).

(3) Since $\langle F, A\rangle \wedge\langle G, B\rangle=\left\{\left\langle x, \min \left\{\mu_{F(\alpha)}(x)\right.\right.\right.$, $\left.\left.\mu_{G(\beta)}(x)\right\}, \max \left\{\gamma_{F(\alpha)}(x), \gamma_{G(\beta)}(x)\right\}\right\rangle: x \in U,(\alpha, \beta) \in$ $A \times B\}$, by Definition 3.8, we have

$$
\begin{aligned}
\diamond(\langle F, A\rangle & \wedge\langle G, B\rangle) \\
= & \left\{\left\langlex, 1-\max \left\{\gamma_{F(\alpha)}(x), \gamma_{G(\beta)}(x)\right\},\right.\right. \\
& \left.\left.\max \left\{\gamma_{F(\alpha)}(x), \gamma_{G(\beta)}(x)\right\}\right\rangle: x \in U,(\alpha, \beta) \in A \times B\right\} .
\end{aligned}
$$

Since $\diamond\langle F, A\rangle=\left\{\left\langle x, 1-\gamma_{F(\alpha)}(x), \gamma_{F(\alpha)}(x)\right\rangle: x \in\right.$ $U, \alpha \in A\}$ and $\diamond\langle G, B\rangle=\left\{\left\langle x, 1-\gamma_{G(\beta)}(x), \gamma_{G(\beta)}(x)\right\rangle\right.$ : $x \in U, \beta \in B\}$, we have

$$
\begin{aligned}
& \diamond\langle F, A\rangle \wedge \diamond\langle G, B\rangle \\
&=\left\{\left\langlex, \min \left\{1-\gamma_{F(\alpha)}(x), 1-\gamma_{G(\beta)}(x)\right\},\right.\right. \\
&\left.\left.\max \left\{\gamma_{F(\alpha)}(x), \gamma_{G(\beta)}(x)\right\}\right\rangle: x \in U,(\alpha, \beta) \in A \times B\right\} \\
&=\left\{\left\langlex, 1-\max \left\{\mu_{F(\alpha)}(x), \mu_{G(\beta)}(x)\right\},\right.\right. \\
&\left.\left.\max \left\{\gamma_{F(\alpha)}(x), \gamma_{G(\beta)}(x)\right\}\right\rangle: x \in U,(\alpha, \beta) \in A \times B\right\} \\
&= \diamond(\langle F, A\rangle \wedge\langle G, B\rangle) .
\end{aligned}
$$

(4) The proof is similar to that of (3).

\section{Conclusions}

Some extensions of soft set theory such as fuzzy soft set theory, intuitionistic fuzzy soft set theory, interval-valued fuzzy soft set theory, vague soft set theory and generalized intuitionistic fuzzy soft set theory are proposed to handle these types of problem parameters. In this paper, the union, intersection, necessity and possibility operations are defined on the generalized intuitionistic fuzzy soft sets. The basic properties of the operations and their interconnections between each other are also presented and discussed. This work not only provides a significant addition to existing theories for handling uncertainties, but also leads to potential areas of further field research and pertinent applications. To extend our work, further research could be done to study the issues on the parameterization reduction of the generalized intuitionistic fuzzy soft sets, and to explore the applications of using the generalized intuitionistic fuzzy soft set approach to solve real world problems such as decision making, forecasting and data analysis. 


\section{References}

[1] M.I. Ali, F. Feng, X. Liu, W.K. Min and M. Shabir, "On some new operations in soft set theory," Computers and Mathematics with Applications, vol. 57, no. 9, pp. 1547-1553, 2009.

[2] Z. Kong, L. Gao and L. Wang, "Comment on "A fuzzy soft set theoretic approach to decision making problems"," Journal of Computational and Applied Mathematics, vol. 223, no. 2, pp. 540-542, 2009.

[3] P.K. Maji, R. Biswas and A.R. Roy, "Fuzzy soft sets," Journal of Fuzzy Mathematics, vol. 9, no. 3, pp. 589-602, 2001.

[4] P.K. Maji, R. Biswas and A.R. Roy, "Intuitionistic fuzzy soft sets," Journal of Fuzzy Mathematics, vol. 9, no. 3, pp. 677-692, 2001.

[5] P.K. Maji, R. Biswas and A.R. Roy, "Soft set theory," Computers and Mathematics with Applications, vol. 45, no. 4-5, pp. 555-562, 2003.

[6] P.K. Maji, A.R. Roy and R. Biswas, "On intuitionistic fuzzy soft sets," Journal of Fuzzy Mathematics, vol. 12, no. 3, pp. 669-683, 2004.

[7] P.K. Maji, More on intuitionistic fuzzy soft sets. In: H. Sakai, M.K. Chakraborty, A.E. Hassanien, D. Slezak and W. Zhu, Editors, Proceedings of the 12th International Conference on Rough Sets, Fuzzy Sets, Data Mining and Granular Computing RSFDGrC 2009, Lecture Notes in Computer Science, vol. 5908, pp. 231-240, 2009.

[8] P. Majumdar and S.K. Samanta, "Generalised fuzzy soft sets," Computers and Mathematics with Applications, vol. 59, no. 4, pp. 1425-1432, 2010.

[9] D. Molodtsov, "Soft set theory - first results," Computers and Mathematics with Applications, vol. 37, no. 4-5, pp. 19-31, 1999.
[10] T.K. Mondal and S.K. Samanta, "Generalized intuitionistic fuzzy sets," Journal of Fuzzy Mathematics, vol. 10, pp. 839-861, 2002.

[11] J.H. Park, K.M. Lim and B.Y. Lee, "Correlation coefficient between generalized Atanassov's intuitionistic fuzzy sets," International Journal of Uncertainty, Fuzziness \& Knowledge-Based Systems, vol. 17, no. 3, pp. 449-461, 2009.

[12] J.H. Park, Y.C. Kwun and J.S. Hwang, "Generalized intuitionistic fuzzy soft sets," Journal of Korean Institute of Intelligent Systems, vol. 21, no. 3, pp. 389394, 2011.

[13] J.H. Park, Y.C. Kwun and M.J. Son, “A generalized intuitionistic fuzzy soft set theoretic approach to decision making problems," International Journal of Fuzzy Logic and Intelligent Systems, vol. 11, no. 2, pp. 71-76, 2011.

[14] A.R. Roy and P.K. Maji, "A fuzzy soft set theoretic approach to decision making problems," Journal of Computational and Applied Mathematics, vol. 203, no. 2, pp. 412-418, 2007.

[15] Y. Zou and Z. Xiao, "Data analysis approaches of soft sets under incomplete information," Knowledge-Based Systems, vol. 21, no. 8, pp. 941-945, 2008.

[16] W. Xu, J. Ma, S. Wang and G. Hao, "Vague soft sets and their properties," Computers and Mathematics with Applications, vol. 59, no. 2, pp. 787-794, 2010.

\section{Jin Han Park}

Professor of Pukyong National University

Research Area: Decision Making, Fuzzy Mathematical Theory, General Topology

E-mail: jihpark@pknu.ac.kr 